\title{
Quantitative Assessment of Posterior Maxillary Arch for Orthodontic Mini-screw Insertion Using Cone Beam Computed Tomography- a Cross Sectional Analysis
}

\section{Solmaz Valizadeh}

Shahid Beheshti University of Medical Sciences School of Dentistry

Abdolhamid Zafarmand

Shahid Beheshti University of Medical Sciences School of Dentistry

Sara Hasanyazdi

Shahid Beheshti University of Medical Sciences School of Dentistry

Korosh Majidi

Royal College of Surgeons: Royal College of Surgeons of England

Mitra Ghazizadeh Ahsaie ( $\square$ mitraghazizadeh@gmail.com )

Shahid Beheshti University of Medical Sciences https://orcid.org/0000-0001-7028-748X

\section{Research}

Keywords: Cone Beam Computed Tomography, Mini-screw, Maxilla, Inter-radicular distance

Posted Date: October 18th, 2021

DOI: https://doi.org/10.21203/rs.3.rs-966820/v1

License: (c) (1) This work is licensed under a Creative Commons Attribution 4.0 International License.

Read Full License 


\section{Abstract}

Background and aim

The aim of this study is to assess the cortical bone thickness and inter-radicular distance between posterior maxillary teeth using Cone Beam Computed Tomography (CBCT).

Methods and material

Cone beam computed tomography records of 35 patients (70 quadrants) from maxilla were evaluated. The images were analyzed using NNT viewer Software (Version 23). The measurements were made on axial sections at 2, 4, 6 and $8 \mathrm{~mm}$ from the CEJ. The optimal sites were defined in terms of (a) mesiodistal palatal or buccal inter-radicular distance, (b) alveolar cortical bone thickness and (c) palatal or buccal safe depth of bone for mini-screw insertion. Descriptive statistics, paired T-test and repeated measure ANOVA were used to analyze the data.

Results

The mean buccal inter-radicular distance was lowest between first and second molar ( $2.44 \mathrm{~mm})$ and highest between first and second premolar $(3.28 \mathrm{~mm})$. The mean palatal inter-radicular distance was lowest between first and second premolar $(3.64 \mathrm{~mm})$ and highest between second premolar and first molar $(5.30 \mathrm{~mm})$. The mean buccal safe depth was lowest between canine and first premolar $(1.96 \mathrm{~mm})$ and highest between first and second molar $(2.61 \mathrm{~mm})$. The mean palatal safe depth was lowest between second premolar and first molar $(3.35 \mathrm{~mm})$ and highest between first and second molar (3.56 $\mathrm{mm}$ ). The thinnest and thickest buccal cortical thickness was detected on canine and first molar (1.04 $\mathrm{mm}$ ) and on the second premolar and second molar $(1.56 \mathrm{~mm})$.

\section{Conclusion}

The quantity and quality of the maxillary alveolar process is an important factor to decide where to insert the orthodontic mini screws, necessitating careful preoperative evaluation.

\section{Introduction}

Orthodontic mini-screws also known as micro-screws, mini-implants or TADs (temporary anchoring devices) are intraoral devices specifically designed to be mounted within the bones of jaw in order to provide absolute anchorage(1). Not needing patient's compliance, today, this technique is widely being used due to its high clinical efficacy, ease of insertion, low cost and reduction of unwanted movement of other teeth $(2,3)$. Application of these devices are, mesialization, distalization, extrusion or intrusion of teeth, space opening and in some cases alignment of midline or inclined plane $(4,5)$. Mini- screws are biocompatible, mostly made of titanium or stainless steel. Resistant to load and corrosion, these devices have various length and diameter which could be chosen according to the available space at the site of insertion $(6,7)$. The decision on where to place the mini-screw depends on the patients' situation and 
orthodontic treatment plan. Mini-screws can be inserted in alveolar part of the maxilla and the mandible between the roots of the tooth of posterior to them, in palate, chin or under the nasal floor $(8,9)$.

Various studies have mentioned complications in applications of mini-screws $(6,10,11)$. Lack of initial stability in bone is one of the main reasons for mini-screw failures. This could be due to insufficient attention to the quality and quantity of bone at the site of insertion and weak bone-screw interface. In addition, improper angle or location of mini-screw placement may result in contact to tooth root, root perforation and sinus floor perforation. Inadequate support from bone around the mini-screws can result in insufficient osseo-integration and lack of stability.

In order to insert implant fixtures into the posterior region of maxilla, a thorough clinical examination and radiographic assessments are needed. These examinations help to assess the quality and quantity of bone and the available space between roots. Conventional radiography such as periapical radiography and panoramic view, can present a general information regarding the selected anchorage site (12). In the study of Tepedino et al(7)., the average interradicular distance was measured using Periapical radiographs. However, due to the two dimensional nature of conventional radiographs, these assessments could be mistaken especially in cases of crowding, dilacerations and superimpositions. Cone beam computed tomography (CBCT) is a three dimensional radiography which can accurately define the shape, morphology and quantity of maxillofacial bone (13-15). This imaging modality presents cross sectional views which can exactly indicate ridge height and width, concavity, angulation degree, the location and shape of tooth root, distance to anatomic landmarks and bone density $(3,6)$. Using CBCT, Haddad et al., indicated higher prevalence of mini-screw failure within posterior maxilla due to reduced cortical bone thickness, and suggested more apical insertion of mini-screw in order to access a denser bucco-lingual and palatal bone level. (16).

This study was aimed at determining the optimal sites of mini-screw insertion in the buccal and palatal alveolar cortical bone in posterior maxilla by determining cortical bone thickness, inter-radicular distance and safe depth of mini-screw insertion using CBCT Imaging.

\section{Materials And Methods}

This was a retrospective cross-sectional study. The study protocol was approved by the institutional review board of Shahid Beheshti University of Medical Sciences (code no: IR.SBMU.DRC.REC.1398.130), and it was conducted in accordance with the Declaration of Helsinki and its subsequent revisions. The study was conducted in accordance with the STROBE statement.

\section{Sample size:}

According to the results of a previous studies $(11,16)$, the sample size was calculated to be 70 CBCT scans, using the following formula: $(z=1.96, d=0.25, S D=1.09)$ 


$$
n=\frac{\left(z^{2} S D^{2}\right)}{d^{2}} \quad n=\frac{(1.96)^{2}(1.09)^{2}}{(25 \%)^{2}} \approx 70
$$

Due to the retrospective nature of the study, the selection bias was an issue. To minimize the effect of selection bias, 700 CBCT scans were assessed for eligibility; out of which, 70 were selected according to the eligibility criteria. Demographic information including age and gender were also recorded for each case.

\section{Evaluation of CT scans:}

This study was conducted on CBCT scans retrieved from the archives of one central oral and maxillofacial radiology clinic in Tehran, Iran. The CBCT scans acquisition was done by New Tom VGI CBCT scanner (Quantitative radiology, Verona, Italy) with the exposure settings of $110 \mathrm{kVp}$ and 3.3-20 $\mathrm{mA}$. The size of field of view was determined according to the patients' size and referral reason. Images were evaluated with the maxillary occlusal plane parallel to the horizontal axis using NNT viewer application (version 23).

Inclusion criteria:

-Dentate patients between 19 to 45 years old, with CBCT scan orders of complete maxillary arch from 2018 to 2020

-Patients must be edentulous in canine, premolars and molars areas.

-Images must have been of adequate resolution/diagnostic quality.

\section{Exclusion Criteria:}

- Any scan that did not satisfy any of the requirements listed in the inclusion criteria.

-edentulous patients

-Presence of severe root dilacerations and anomalies

-Presence of periodontal disease and bone defects

-Any scan that included maxillofacial trauma, orthognathic surgery, history of orthodontic treatment congenital anomalies or pathologies such as cysts or tumors, impacted teeth, intraoral exostoses, dental implant or bone graft.

- Patients receiving medications affecting the bone metabolism such as bisphosphonate drug, osteoporosis or other diseases affecting the bone quality and quantity 
CBCT scans were evaluated by a calibrated oral and maxillofacial radiologist with 20 years of clinical experience. The primary outcome of this study was to assess the bone quantity of different parts of the posterior maxilla for cortical bone thickness and inter-radicular distance. For this reason, the following parameters were separately measured for ten teeth (canine to second molar at both sides), using NNT Viewer software with a ruler with $0.1 \mathrm{~mm}$ accuracy. There were no restrictions with respect to the use of image enhancement filters. The variables were all measured in millimeters. From each CBCT scan, axial CBCT sections at the level of CEJ was detected. The following measurements were performed at 2, 4, 6 and $8 \mathrm{~mm}$ vertical distance from the CEJ:

1- Distance between buccal roots: The shortest distance between buccal roots of premolars and molars (Figure-2a). As canine tooth has one root, the distance between root canine and buccal root of first premolar was measured in this category.

2- Distance between palatal roots: The shortest distance between Palatal roots of premolars and molars (Figure-2b).

3- Buccal cortex thickness: Distance between outer buccal cortex to buccal roots of canine, premolars and molars (Figure-2c).

4- Safe buccal depth: The distance between outer cortex of buccal bone to the narrowest point between buccal roots of premolars and molars (Figure-2d).

5- Safe palatal depth: The distance between outer cortex of palatal bone to the narrowest point between palatal roots of premolars and molars (Figure-2e).

Subjectivity of measurement was another source of bias in this study. To verify the reliability of measurements, the intra-observer agreement was calculated, using the Intra-class coefficient (ICC) test. For this purpose, 20 CBCT scans were evaluated twice with a 1-month interval.

Statistical analysis:

All data were entered into a database system and evaluated using SPSS ${ }^{8}$ for Windows version 21(SPSS Inc., Chicago, IL, USA, 2012). Patients' data were analyzed anonymously. Every case was assigned a registration number before evaluation to allow explicit and anonymous attribution of necessary information. Data analysis was performed with descriptive statistics, paired T-test and repeated measure ANOVA. The level of significance was set at $p=0.05$.

\section{Results}

\section{Intra-Operator Reliability}

Measures for the first and second replicates of 20 patients were recorded and intra-class correlation coefficients (ICC) were established for all measurements. Most measures demonstrated a high degree of 
reliability between the first and second replicates with ICC values exceeding from 0.81 to 0.98 .

\section{Quantitative Assessments}

A total of 350 sites were assessed in both left and right sides of posterior maxilla.

\section{Distance between buccal and palatal roots (Figure-3 A to D):}

The mean buccal inter-radicular distance generally increased from $2 \mathrm{~mm}$ from CEJ to $6 \mathrm{~mm}$ from CEJ and further decreased from $6 \mathrm{~mm}$ from CEJ to $8 \mathrm{~mm}$ from CEJ. The mean buccal inter-radicular distance between canine and first premolar were $2.52 \pm 0.86,2.72 \pm 0.81,2.87 \pm 0.90$ and $2.73 \pm 1.38$ at 2, 4, 6 and $8 \mathrm{~mm}$ from CEJ. The mean buccal inter-radicular distance between first and second premolar were $2.75 \pm$ $0.83,3.01 \pm 0.80,3.28 \pm 0.94$ and $2.77 \pm 1.50$ at $2,4,6$ and $8 \mathrm{~mm}$ from CEJ. The mean buccal interradicular distance between second premolar and first molar were $2.71 \pm 0.80,2.79 \pm 0.84,2.87 \pm 0.94$ and $2.78 \pm 1.57$ at $2,4,6$ and $8 \mathrm{~mm}$ from CEJ. The mean buccal inter-radicular distance between first molar and second molar were $2.36 \pm 0.91,2.44 \pm 0.85,2.00 \pm 1.09$ and $1.64 \pm 1.11$ at 2, 4, 6 and $8 \mathrm{~mm}$ from CEJ (Table-1).

The mean palatal inter-radicular distance generally increased from $2 \mathrm{~mm}$ from CEJ to $8 \mathrm{~mm}$ from CEJ and were higher than buccal inter-radicular distances. The mean buccal inter-radicular distance between first and second premolar were $3.10 \pm 0.59,3.46 \pm 0.69,3.48 \pm 0.89$ and $3.64 \pm 0.81$ at $2,4,6$ and $8 \mathrm{~mm}$ from CEJ. The mean buccal inter-radicular distance between second premolar and first molar were $3.68 \pm 0.90$, $4.40 \pm 1.09,5.01 \pm 1.08$ and $5.30 \pm 1.08$ at 2, 4, 6 and $8 \mathrm{~mm}$ from CEJ. The mean buccal inter-radicular distance between first molar and second molar were $2.92 \pm 0.99,3.52 \pm 0.94,3.88 \pm 1.17$ and $4.15 \pm 0.95$ at 2, 4, 6 and $8 \mathrm{~mm}$ from CEJ (Table-1). 


\begin{tabular}{|c|c|c|c|c|c|c|c|c|}
\hline \multicolumn{9}{|c|}{ Distance between buccal roots } \\
\hline Distance from CEJ & $2 \mathrm{~mm}$ & & $4 \mathrm{~mm}$ & & $6 \mathrm{~mm}$ & & $8 \mathrm{~mm}$ & \\
\hline Teeth & mean & SD & mean & SD & mean & SD & mean & SD \\
\hline Canine- $1^{\text {st }} P$ & 2.52 & 0.86 & 2.72 & 0.81 & 2.87 & 0.90 & 2.73 & 1.38 \\
\hline $1^{\text {st }} P-2^{\text {nd }} P$ & 2.75 & 0.83 & 3.01 & 0.80 & 3.28 & 0.94 & 2.77 & 1.50 \\
\hline $2^{\text {nd }} P-1^{\text {st }} M$ & 2.71 & 0.80 & 2.79 & 0.84 & 2.87 & 0.94 & 2.78 & 1.15 \\
\hline $1^{\text {st }} M-2^{\text {nd }} M$ & 2.36 & 0.91 & 2.44 & 0.85 & 2.02 & 1.09 & 1.64 & 1.11 \\
\hline \multicolumn{9}{|c|}{ Distance between palatal roots } \\
\hline Distance from CEJ & $2 \mathrm{~mm}$ & & $4 \mathrm{~mm}$ & & $6 \mathrm{~mm}$ & & $8 \mathrm{~mm}$ & \\
\hline Teeth & mean & SD & mean & SD & mean & SD & mean & SD \\
\hline $1^{\text {st }} P-2^{\text {nd }} P$ & 3.10 & 0.59 & 3.46 & 0.69 & 3.48 & 0.89 & 3.64 & 0.81 \\
\hline $2^{\text {nd }} P-1^{\text {st }} M$ & 3.68 & 0.90 & 4.40 & 1.09 & 5.01 & 1.08 & 5.30 & 1.03 \\
\hline $1^{\text {st }} M-2^{\text {nd }} M$ & 2.92 & 0.99 & 3.52 & 0.94 & 3.88 & 1.17 & 4.15 & 0.95 \\
\hline
\end{tabular}

Table-1: Distance between buccal and palatal roots in posterior maxilla in millimeter. $\mathrm{M}=$ Molar, $\mathrm{mm}=$ millimeter, $\mathrm{P}=$ premolar, $\mathrm{SD}=$ Standard Deviation,

\section{Buccal cortical thickness (Figure-3 E and F):}

The mean buccal cortex thickness increased from $2 \mathrm{~mm}$ to $6 \mathrm{~mm}$ CEJ and further decreased from $6 \mathrm{~mm}$ to $8 \mathrm{~mm}$ CEJ on all posterior teeth in maxilla. The thickness of buccal cortex was highest on second premolar tooth and was lowest on first molar (Table-2)

\begin{tabular}{|c|c|c|c|c|c|c|c|c|}
\hline \multicolumn{9}{|c|}{ Buccal cortical bone thickness } \\
\hline Distance from CEJ & $2 \mathrm{~mm}$ & & $4 \mathrm{~mm}$ & & $6 \mathrm{~mm}$ & & $8 \mathrm{~mm}$ & \\
\hline Teeth & mean & SD & mean & SD & mean & SD & mean & SD \\
\hline Canine & 0.76 & 0.31 & 1.04 & 0.44 & 0.80 & 0.54 & 0.70 & 0.50 \\
\hline $1^{\text {st }} P$ & 0.58 & 0.53 & 0.86 & 0.52 & 0.61 & 0.41 & 0.52 & 0.37 \\
\hline $2^{\text {nd }} P$ & 1.05 & 0.96 & 1.56 & 0.81 & 1.23 & 0.71 & 0.95 & 0.77 \\
\hline $1^{\text {st }} M$ & 0.46 & 0.54 & 0.71 & 0.46 & 0.53 & 0.53 & 0.47 & 0.49 \\
\hline $2^{\text {nd }} M$ & 0.78 & 0.42 & 1.54 & 0.70 & 1.33 & 0.80 & 1.19 & 0.96 \\
\hline
\end{tabular}


Table-2: buccal cortical bone thickness in millimeter.

\section{Safe buccal and palatal depth (Figure-3 G to J):}

The safe buccal depth increased from $2 \mathrm{~mm}$ to $8 \mathrm{~mm}$ from CEJ from canine to second molar. The mean safe buccal depth between canine and first premolar were 1.62 $\pm 0.69,1.96 \pm 0.70,1.72 \pm 0.65$ and $1.56 \pm$ 0.74 at 2, 4, 6 and $8 \mathrm{~mm}$ from CEJ. The safe buccal depth between first premolar and second premolar were $2.24 \pm 1.10,2.52 \pm 0.84,2.11 \pm 0.76$ and $1.81 \pm 0.76$ at $2,4,6$ and $8 \mathrm{~mm}$ from CEJ. The mean safe buccal depth between second premolar and first molar were $2.37 \pm 0.93,2.59 \pm 0.81,2.16 \pm 0.80$ and 2.06 \pm 0.79 at $2,4,6$ and $8 \mathrm{~mm}$ from CEJ. The mean safe buccal depth between first molar and second molar were $1.63 \pm 0.99,2.611 .03,2.69 \pm 0.86$ and $2.45 \pm 0.82$ at $2,4,6$ and $8 \mathrm{~mm}$ from CEJ The highest buccal depth was detected between first and second molar at $4 \mathrm{~mm}$ depth from CEJ (Table-3).

The safe palatal depth increased from $2 \mathrm{~mm}$ to $8 \mathrm{~mm}$ from CEJ from first premolar to second molar. The mean safe buccal depth between first and second premolar were 1.83 $\pm 1.6,2.42 \pm 0.72,2.9 \pm 0.81$ and $3.61 \pm 0.70$ at 2, 4, 6 and $8 \mathrm{~mm}$ from CEJ. The safe palatal depth between second premolar and first molar were $1.88 \pm 0.88,2.37 \pm 0.80,2.76 \pm 0.83$ and $3.35 \pm 0.87$ at $2,4,6$ and $8 \mathrm{~mm}$ from CEJ. The mean safe palatal depth between first molar and second molar were $2.01 \pm 1.05,2.82 \pm 0.89,3.00 \pm 0.77$ and $3.56 \pm 0.72$ at 2, 4, 6 and $8 \mathrm{~mm}$ from CEJ. The highest palatal depth was detected between first and second molar at $8 \mathrm{~mm}$ depth from CEJ (Table-3).

\begin{tabular}{|c|c|c|c|c|c|c|c|c|}
\hline \multicolumn{9}{|l|}{ Safe buccal depth } \\
\hline Distance from CEJ & $2 \mathrm{~mm}$ & & $4 \mathrm{~mm}$ & & $6 \mathrm{~mm}$ & & $8 \mathrm{~mm}$ & \\
\hline Teeth & mean & SD & mean & SD & mean & SD & mean & SD \\
\hline Canine- $1^{\text {st }} \mathrm{P}$ & 1.62 & 0.69 & 1.96 & 0.70 & 1.72 & 1.80 & 1.56 & 1.50 \\
\hline $1^{\text {st }} P-2^{\text {nd }} P$ & 2.24 & 1.10 & 2.52 & 0.84 & 2.11 & 2.30 & 1.81 & 1.90 \\
\hline $2^{\text {nd }} P-1^{\text {st }} M$ & 2.37 & 0.93 & 2.59 & 0.81 & 2.16 & 2.15 & 2.06 & 2.00 \\
\hline $1^{\text {st }} M-2^{\text {nd }} M$ & 2.56 & 0.80 & 2.61 & 1.03 & 2.50 & 2.50 & 2.45 & 2.50 \\
\hline \multicolumn{9}{|l|}{ Safe palatal depth } \\
\hline Distance from CEJ & $2 \mathrm{~mm}$ & & $4 \mathrm{~mm}$ & & $6 \mathrm{~mm}$ & & $8 \mathrm{~mm}$ & \\
\hline Teeth & mean & SD & mean & SD & mean & SD & mean & SD \\
\hline $1^{\text {st }} P-2^{\text {nd }} P$ & 1.83 & 1.06 & 2.42 & 0.72 & 2.90 & 0.81 & 3.61 & 0.70 \\
\hline $2^{\text {nd }} P-1^{\text {st }} M$ & 1.88 & 0.88 & 2.37 & 0.80 & 2.76 & 0.83 & 3.35 & 0.87 \\
\hline $1^{\text {st }} M-2^{\text {nd }} M$ & 2.01 & 1.05 & 2.82 & 0.89 & 3.00 & 0.77 & 3.56 & 0.72 \\
\hline
\end{tabular}


Table-3: Safe buccal and palatal bone thickness in millimeter.

\section{Discussion}

The aim of this study was to assess the optimal sites of mini-screw insertion in the buccal and palatal alveolar cortical bone in posterior maxilla by determining cortical bone thickness, inter-radicular distance and safe depth of mini-screw insertion using CBCT Imaging.

Temporary anchorage devices such as mini- screws are absolute anchors placed in jaw bone enabling dental movement in orthodontic treatments. In the study of Zheng et al., it was indicated that the success rate of mini-screws could reach up to $80 \%$ (1) especially when movement of molar teeth is needed, however it is strongly recommended to assess the quality and quantity of bone prior to screw insertion. Previous studies have shown $20 \%$ injury rate during mini-screw insertion due to inaccuracy of insertion and lack of surgical guidance $(17,18)$

Watanabe et al., indicated that maxillary mini-screws are significantly more stable than mandibular screws (19), in addition, they indicated that the distance between screw and root was significantly lower in failure groups. The results of this study indicated that in the buccal region, increase and a subsequent decrease in inter-radicular space from 2 to $8 \mathrm{~mm}$ distance to CEJ in posterior maxilla were detected. The most distant inter-radicular space was detected between second premolar and first molar at $6 \mathrm{~mm}$ distance to CEJ. This result is in consensus with the study of Yoon et al.,(20). Al Amiri MS et al., also evaluated optimal position of orthodontic mini-screws in the maxilla. Their results indicated that buccally, the interdental bone depth was significantly greater between the second premolar and first molar(21). However, in the study of Liu et al, the best segment for mini-screw insertion was assumed between first and second molar (22). In this study, the least distance between palatal roots was detected at $2 \mathrm{~mm}$ from CEJ between first molar and second molar, however at 4, 6 and $8 \mathrm{~mm}$ from CEJ, the distance between first premolar and second premolar teeth was lowest. In another study, mini-screw implantation guided by stereolithographic surgical stent based on CBCT derived 3D images were performed. The results suggested no root damage in the stent group. However, without a surgical stent, four cases out of 10 mini-screws contacted tooth roots. This study concluded that when facing limited inter-radicular distance, multiple impacted teeth and extended maxillary sinus, CBCT guided techniques is strongly suggested (17). Liu et al., investigated the anatomy of the mandibular buccal shelf with CBCT to assess appropriate region of miniscrew insertion for distalization of mandibular dentition $(22,23)$ the results suggested buccal alveolar bone thickness increase from premolar to the molar and from crest to the mandibular roots. The buccal inter-radicular spaces were smallest between mesiodistal roots of the second molar.

Marquezan et al indicated that cortical bone thickness can influence primary mini screw stability (24). In this study, cortical bone thickness was highest on second premolar at 2 to $6 \mathrm{~mm}$ from CEJ and further on second molar from 6 to $8 \mathrm{~mm}$ from CEJ. Thinner cortical bone may negatively influence the primary screw stability. However, the intra bony depth is another influencing factor affecting primary stability of 
mini screws. In this study, the highest buccal and palatal depth is detected between first molar and second molar. Jin et al., indicated that regardless of the cortical bone density, more energy was required to remove the miniscrews as the implantation depth increased, indicating higher resistance and less risk of falling out (10).

\section{Conclusion}

The highest distance between buccal and palatal roots of posterior maxilla was detected between first and second premolars at $6 \mathrm{~mm}$ distance from the CEJ and between second premolar and first molar at 8 $\mathrm{mm}$ distance from CEJ respectively. Therefore it can be assumed that the most optimal sites for miniscrew insertion in maxilla is nearly at the mid root site of premolar-molar area.

\section{Limitations and suggestions:}

It is important to know that the thickness of the gingival soft tissue may also affect the prognosis of the mini-screws therefore further studies are recommended to take this factor into account. Future studies may also evaluate larger sample sizes and assess the relationship between systemic diseases, drug consumption, aging and craniofacial anomalies on quantitative assessments of both maxilla and mandible arches.

\section{Declarations}

\section{Availability of data and materials:}

The datasets used and/or analyzed during the current study are available from the corresponding author on reasonable request.

\section{Acknowledgements:}

The authors would like to thank Dr. Mahshid Namdari for her great efforts in statistical analysis.

\section{Funding:}

This research received no specific grant from any funding agencies.

\section{Affiliations}

\section{Department of Oral and Maxillofacial Radiology, School of Dentistry, Shahid Beheshti University of Medical Sciences, Tehran, Iran}

Solmaz Valizadeh \& Mitra Ghazizadeh Ahsaie 
2. Department of Orthoodontics, School of Dentistry, Shahid Beheshti University of Medical Sciences, Tehran, Iran

Abdolhamid Zafarmand

3. School of Dentistry, Shahid Beheshti University of Medical Sciences, Tehran, Iran

Sarah Hasanyazdi

4. DDS,DipDCSc, Edingburgh, England

Korosh Majidi

\section{Contributions}

Valizadeh S. and Zafarmand A designed and supervised the study process. Hassanyazdi S performed data acquisition and investigation. Valizadeh $S$ and Hassan yazdi $S$ participated in the data analysis and interpretation. Ghazizadeh Ahsaie and Majidi drafted the manuscript. All the authors have reviewed and approved the final article.

\section{Corresponding author}

Correspondence to Mitra Ghazizadeh Ahsaie

\section{Ethics approval and consent to participate}

The study protocol was approved by the institutional review board of Shahid Beheshti University of Medical Sciences (code no: IR.SBMU.DRC.REC.1398.130).

\section{Consent for publication}

All authors consent to publication of this manuscript.

\section{Competing interests}

The authors declare that they have no competing interests.

\section{References}

1. Zheng X, Sun Y, Zhang Y, Cai T, Sun F, Lin J. Implants for orthodontic anchorage: An overview. Medicine. 2018;97(13). 
2. Suteerapongpun P, Wattanachai T, Janhom A, Tripuwabhrut P, Jotikasthira D. Quantitative evaluation of palatal bone thickness in patients with normal and open vertical skeletal configurations using cone-beam computed tomography. Imaging science in dentistry. 2018;48(1):51-7.

3. Marchi A, Camporesi M, Festa M, Salvatierra L, Izadi S, Farronato G. Drilling Capability of Orthodontic Miniscrews: In Vitro Study. Dentistry Journal. 2020;8(4):138.

4. Alharbi F, Almuzian M, Bearn D. Anchorage effectiveness of orthodontic miniscrews compared to headgear and transpalatal arches: a systematic review and meta-analysis. Acta Odontologica Scandinavica. 2019;77(2):88-98.

5. Elshebiny T, Palomo JM, Baumgaertel S. Anatomic assessment of the mandibular buccal shelf for miniscrew insertion in white patients. American Journal of Orthodontics and Dentofacial Orthopedics. 2018;153(4):505-11.

6. Lee DW, Park JH, Bay RC, Choi SK, Chae JM. Cortical bone thickness and bone density effects on miniscrew success rates: A systematic review and meta-analysis. Orthodontics \& Craniofacial Research. 2021;24:92-102.

7. Tepedino M, Cattaneo PM, Masedu F, Chimenti C. Average interradicular sites for miniscrew insertion: should dental crowding be considered? Dental press journal of orthodontics. 2017;22:90-7.

8. Aksoy U, Aksoy S, Orhan K. A cone-beam computed tomography study of the anatomical relationships between mandibular teeth and the mandibular canal, with a review of the current literature. Microscopy research and technique. 2018;81(3):308-14.

9. Casaña-Ruiz MD, Bellot-Arcís C, Paredes-Gallardo V, García-Sanz V, Almerich-Silla JM, MontielCompany JM. Risk factors for orthodontic mini-implants in skeletal anchorage biological stability: a systematic literature review and meta-analysis. Scientific reports. 2020;10(1):1-10.

10. Jin J, Kim G-T, Kwon J-S, Choi S-H. Effects of intrabony length and cortical bone density on the primary stability of orthodontic miniscrews. Materials. 2020;13(24):5615.

11. Mallick S, Murali P, Kuttappa M, Shetty P, Nair A. Optimal sites for mini-implant insertion in the lingual or palatal alveolar cortical bone as assessed by cone beam computed tomography in South Indian population. Orthodontics \& Craniofacial Research. 2021;24(1):121-9.

12. Shelley AM, Glenny A-M, Goodwin M, Brunton P, Horner K. Conventional radiography and crosssectional imaging when planning dental implants in the anterior edentulous mandible to support an overdenture: a systematic review. Dentomaxillofacial Radiology. 2013;43(2):20130321.

13. Shelley A, Ferrero A, Brunton P, Goodwin M, Horner K. The impact of CBCT imaging when placing dental implants in the anterior edentulous mandible: a before-after study. Dentomaxillofacial Radiology. 2015;44(4):20140316.

Page 12/17 
14. Moshfeghi M, Rahimi H, Rahimi H, Nouri M, Bagheban AA. Predicting mandibular growth increment on the basis of cervical vertebral dimensions in Iranian girls. Progress in orthodontics. 2013;14(1):1-6.

15. Safi Y, Moshfeghi M, Rahimian S, Kheirkhahi M, Eslami Manouchehri M. Assessment of nasopalatine canal anatomic variations using cone beam computed tomography in a group of Iranian population. Iranian Journal of Radiology. 2017;14(1).

16. Haddad R, Saadeh M. Distance to alveolar crestal bone: a critical factor in the success of orthodontic mini-implants. Progress in orthodontics. 2019;20(1):1-7.

17. Qiu L, Haruyama N, Suzuki S, Yamada D, Obayashi N, Kurabayashi T, et al. Accuracy of orthodontic miniscrew implantation guided by stereolithographic surgical stent based on cone-beam CTderived 3D images. The Angle Orthodontist. 2012;82(2):284-93.

18. Lindgren C, Sennerby L, Mordenfeld A, Hallman M. Clinical histology of microimplants placed in two different biomaterials. Int J Oral Maxillofac Implants. 2009;24(6):1093-100.

19. Watanabe H, Deguchi T, Hasegawa M, Ito M, Kim S, Takano-Yamamoto T. Orthodontic miniscrew failure rate and root proximity, insertion angle, bone contact length, and bone density. Orthodontics \& craniofacial research. 2013;16(1):44-55.

20. Yoon J-H, Cha J-Y, Choi YJ, Park W-S, Han S-S, Lee K-J. Simulation of miniscrew-root distance available for molar distalization depending on the miniscrew insertion angle and vertical facial type. PloS one. 2020;15(9):e0239759.

21. Al Amri MS, Sabban HM, Alsaggaf DH, Alsulaimani FF, Al-Turki GA, Al-Zahrani MS, et al. Anatomical consideration for optimal position of orthodontic miniscrews in the maxilla: a CBCT appraisal. Annals of Saudi Medicine. 2020;40(4):330-7.

22. Liu H, Wu X, Yang L, Ding Y. Safe zones for miniscrews in maxillary dentition distalization assessed with cone-beam computed tomography. American Journal of Orthodontics and Dentofacial Orthopedics. 2017;151(3):500-6.

23. Liu H, Wu X, Tan J, Li X. Safe regions of miniscrew implantation for distalization of mandibular dentition with CBCT. Progress in orthodontics. 2019;20(1):1-8.

24. Marquezan M, Mattos CT, Sant'Anna EF, de Souza MMG, Maia LC. Does cortical thickness influence the primary stability of miniscrews?: a systematic review and meta-analysis. The Angle Orthodontist. 2014;84(6):1093-103.

\section{Figures}




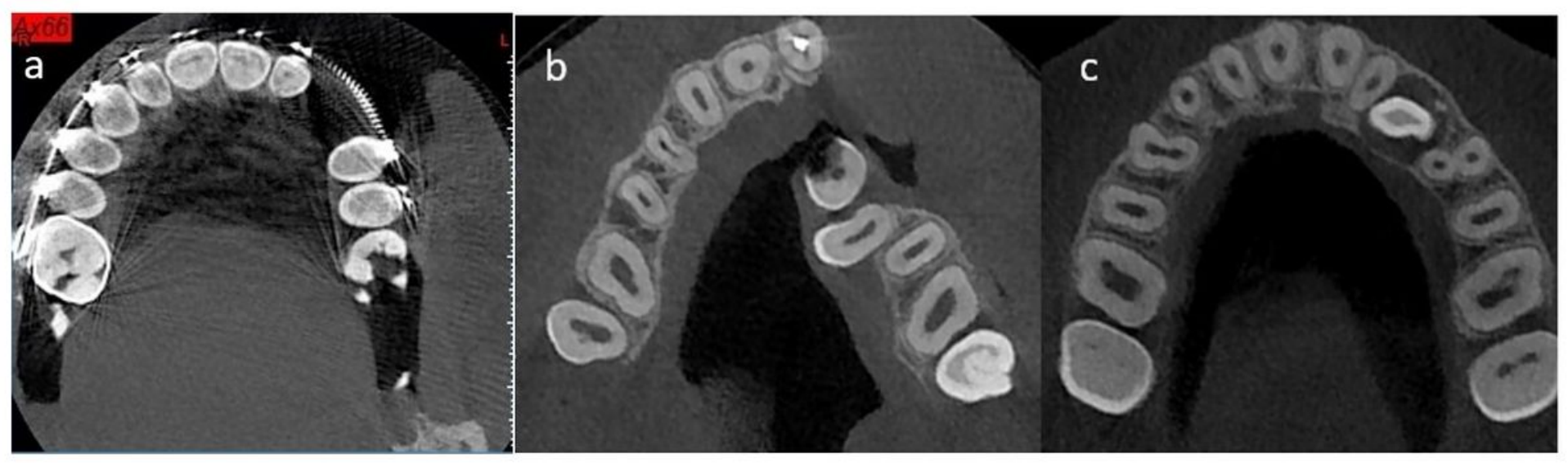

\section{Figure 1}

Example of excluded samples. (a) Orthodontic treatment, (b) cleft palate, (c) impacted canine. 




10

\section{Figure 2}

Axial CBCT scans demonstrating measurement (in millimeter) of inter-radicular distance, cortex thickness and safe depth of mini-screw insertion. (a) Distance between buccal roots, (b) Distance between palatal roots, (c) Buccal cortex thickness, (d) Safe buccal depth and (e) Safe palatal depth. 

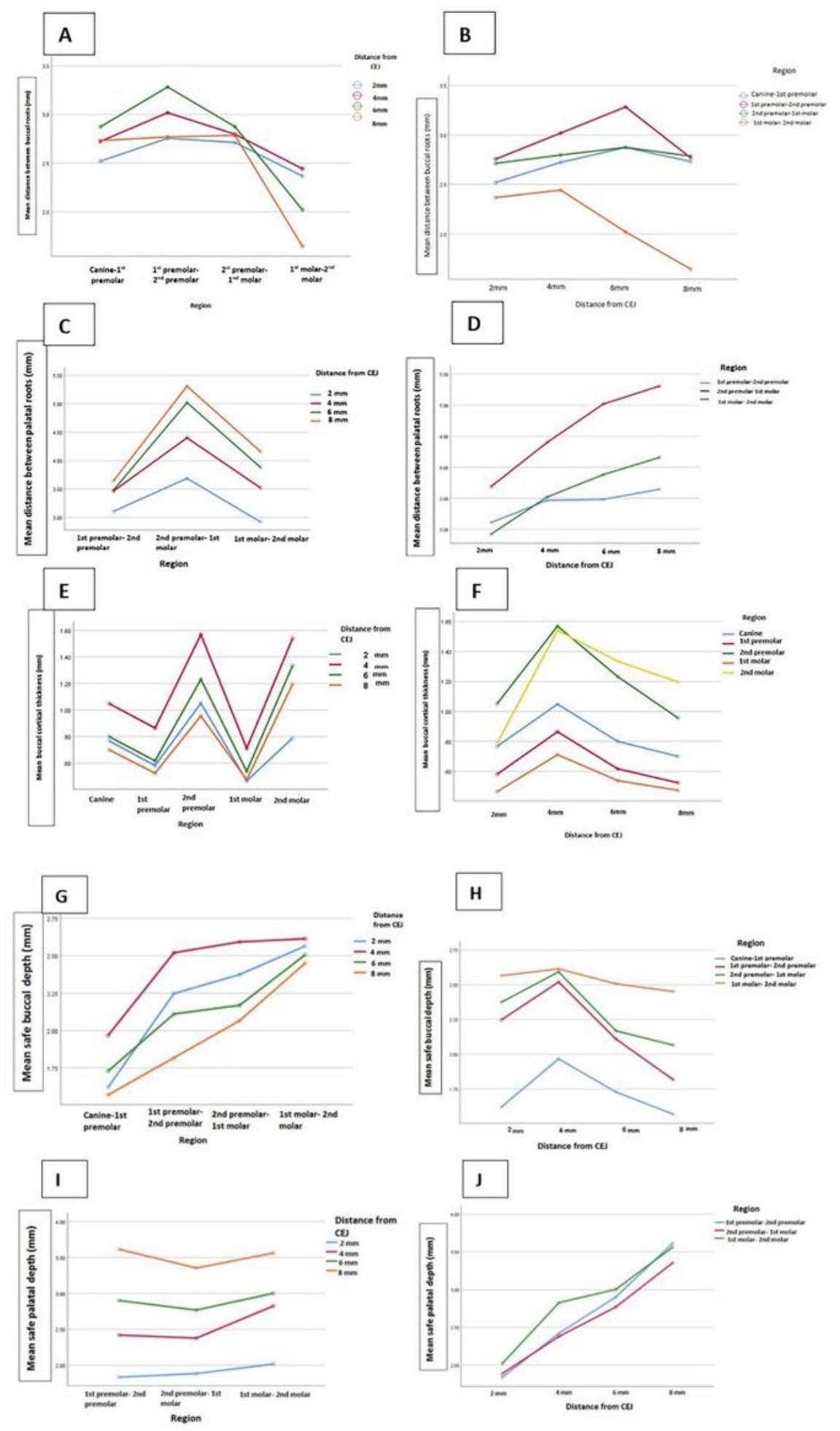

\section{Figure 3}

Graphs indicating (A) mean distance between buccal roots based on tooth region, (B) Mean distance between buccal roots in relation to distance from CEJ,(C) mean distance between palatal roots based on tooth region, (D) mean distance between palatal roots in relation to distance from CEJ, (E) mean buccal cortical bone thickness based on tooth region, $(F)$ mean buccal cortical bone thickness in relation to distance from CEJ, (G) mean buccal depth based on tooth region, $(\mathrm{H})$ mean buccal depth in relation to 
distance from CEJ, (I) mean palatal depth based on tooth region, $(\mathrm{J})$ mean palatal depth in relation to distance from CEJ. 